\title{
Critical challenges in doctoral education: Highlights of the biennial meeting of the International Network for Doctoral Education in Nursing, Tokyo, Japan, 2007
}

\author{
Richard W. REDMAN \\ School of Nursing, University of Michigan, Ann Arbor, Michigan, USA
}

\begin{abstract}
A number of issues and challenges in doctoral nursing education were presented at the biennial meeting of the International Network for Doctoral Education in Nursing in June 2007. The themes addressed included: global health workforce shortages and the aging of the nursing workforce; the need for more nursing doctoral programs; strengthening of the research training in doctoral programs, and the need for increased involvement of nurse leaders in the health and social policy arena.
\end{abstract}

Key words: doctoral education, health workforce, international nursing issues.

\section{INTRODUCTION}

The International Network for Doctoral Education in Nursing (INDEN) is a global membership organization of faculty members and students who are committed to the advancement and promotion of high-quality doctoral education in nursing through national and international collaboration and cooperation. The INDEN began in 1995 and has held international meetings every 2 years since then. Typically, the INDEN holds its meeting adjacent to large international nursing meetings. The most recent meeting was held in late May 2007, in Tokyo, just prior to the International Congress of Nursing (ICN) meetings held in Yokohama, Japan.

The biennial INDEN meetings were held from 26 to 28 May 2007. The meeting was cosponsored by St Luke's College of Nursing and the meetings were held in the College's campus facilities in Tokyo. The title for this biennial meeting was Critical Challenges in Nursing Doctoral Education for the 21st Century. Approximately, 100 faculty members and students from doctoral education programs throughout the world participated in the scientific sessions and the business meetings.

Correspondence: Richard W. Redman, School of Nursing, University of Michigan, 400 North Ingalls Street, Suite 1305, Ann Arbor, MI 48109-0482, USA. Email: rwr@umich.edu Received 30 August 2007; accepted 21 September 2007.
In addition, a poster exhibition of doctoral student research activities was available for viewing throughout the meetings. The program for the scientific program is presented in Appendix I. Summaries of the highlights from the various presentations are presented here.

\section{GLOBAL PERSPECTIVE}

Dr Jean Yan, Chief Scientist for Nursing and Midwifery at the World Health Organization (WHO), provided the context for the meetings by addressing the needs of doctorally prepared nurses globally. She addressed the urgent need of increasing the number of nurses with doctoral preparation who will become involved in addressing the critical workforce shortages through the preparation of nurses for basic and advanced practise, who will generate knowledge and translate the scientific findings to improve the health of citizens throughout the world, and who will engage in health policy.

She presented extensive data on morbidity and mortality and discussed the priority health problems that people face throughout the world. These patterns define essential roles for nurses with doctorates in terms of research programs and the preparation of present and future health-care workers. Although an urgent need exists for increasing the number of doctoral education programs throughout the world, the need for the development and expansion of doctoral programs in WHO 
regions is most urgent in Africa, the eastern Mediterranean area, and South-East Asia.

The involvement of nurse leaders is equally important at the policy-making level. It is essential that nurse leaders be involved in the development of policy that ensures equitable access to a well-prepared health workforce throughout the world. There is an urgent need to scale up the preparation of health workers, as well as to ensure an equitable distribution of that workforce.

The global patterns described by Dr Yan have implications for doctoral education programs. Curricula must ensure that the learning experiences of students examine these global trends and provide mentored experiences that will assist doctoral students in learning about their roles and responsibilities as leaders in their country. In addition, the research that is conducted by our developing scientists must address the priority health needs faced by the population.

\section{REGIONAL PERSPECTIVES}

Developments and challenges in doctoral education in various global regions were then presented. These regional perspectives included sub-Saharan Africa, Australasia, the UK and Europe, Japan, and Thailand.

\section{Sub-Saharan Africa}

Professor Hester Klopper, Dean and Director at NorthWest University in South Africa, presented a brief history of doctoral education in sub-Saharan Africa. Nursing doctoral education is only available in four countries in Africa: Kenya, Namibia, Nigeria, and South Africa. Doctoral education began in South Africa in 1967 and has grown to nine programs. Limited data are available for the African continent, but the South African programs have produced 472 doctoral graduates since 1967 . The demand for doctorally prepared nurses continues to outpace the supply available.

Professor Klopper presented several challenges faced by doctoral education programs in Africa. These include capacity-building, globalization, and career paths. The need for building capacity to educate doctorally prepared nurses is urgent, particularly in light of the resource challenges and the health needs faced on the continent. Capacity-building also is needed in terms of making the curricula in doctoral programs relevant and creating a culture that values and recognizes research. The globalization that is evident in every aspect of lives today has major implications for the flow of information and the preparation of students as global citizens. This globalization also has important implications for creat- ing an international agenda for research, teaching, and service for all disciplines, including nursing. Finally, doctoral education programs must give equal balance to advanced practise, education, research, and management so that graduates are prepared for the challenges they will face in future roles. In addition, graduates must be mentored in ways that will prepare them to influence health and social policy.

\section{Australasia}

Professor Kim Usher, of James Cook University in Australia, discussed the challenges in Australia, New Zealand, and the Western Pacific region. Although the education and health systems in Australia and New Zealand are well-developed, this is not the case in most of the Western Pacific region. Australia and New Zealand are the only countries in the region offering doctoral nursing education. Very limited numbers of nurses in the Pacific Island countries are able to obtain funding and seek doctoral education in those countries where programs are available. There is considerable variation in nursing education in general across the Western Pacific region.

Doctoral nursing programs began in the 1980s in Australia and they tend to follow the British model of mentored research training rather than formal course offerings, as found in programs that follow the US model. There has been pressure on nursing faculty members at undergraduate and Master's degree levels to seek doctoral level preparation and, as a result, many are seeking doctoral studies on a part-time basis while continuing to be employed as academics.

Increasingly, clinical chairs are becoming established in doctoral programs in Australia and New Zealand to develop partnerships between academic and practise settings. Professor Usher reported that seven doctoral programs in Australia and two in New Zealand have established a total of 24 clinical chairs. An additional five chairs are in the planning stages. These chairs provide a strong focus for the development of research programs in doctoral education and also provide a linkage for improving evidence-based practise in the clinical setting.

A final challenge is the number of doctoral graduates who seek employment in non-academic roles and settings. These types of employment roles include entrepreneurial activities, consultancy roles, policy agencies in the public and private sectors, and roles in the department of defense. Although these are important roles and settings for doctorally prepared nurses, it also exagger- 
ates the growing need for doctorally prepared faculty members in academic settings.

\section{UK and Europe}

Professor Anne Marie Rafferty, of King's College, London, presented on doctoral education in the UK and Europe, focusing on the UK as a case study. In the UK, $\mathrm{PhD}$ education for all disciplines was introduced in 1917 at Oxford University and set the stage as the standard qualification for entry into research and academic professions. The demand for doctorally prepared individuals has increased considerably due to demand for the high-level skills needed to contribute to an increasingly sophisticated market economy. In the UK, there has been a $31 \%$ increase in the number of $\mathrm{PhD}$ students between 1999 and 2003 at UK universities to meet this increased demand. In nursing, the number of completed doctoral dissertations in nursing in the UK has increased from a total of 48, completed between 1983 and 1992, to a total of 156, completed between 1993 and 2002.

In Europe, there is rapid growth in doctoral studies in general due to the harmonization underway with the European Union. Education at both the undergraduate and graduate levels across disciplines is seeing some standardization. In addition, there is increasing global competition for doctoral students in all disciplines. Another development is the growth of different types of doctoral degrees, ranging from the mentored research degree to the structured or taught doctorate. There is the $\mathrm{PhD}$ by publication model that is available in some countries. Finally, the professional doctorate, for disciplines such as clinical psychology, engineering, education, and nursing, is growing rapidly. The professional doctorate is a program of advanced study designed to meet the specific needs of practising disciplines and is currently available in 19 disciplines in the UK. The professional doctorate was first introduced for nursing in the UK in 1995 at the University of Ulster in Northern Ireland. Considerable variation is seen in doctoral education, both in the $\mathrm{PhD}$ and the professional doctorate.

New roles are also being seen for doctorally prepared nurses due to advances in technology and health care. Roles such as the consultant practitioner and clinical researcher are in demand. In addition, the need for traditional academic roles continues as the need for an increasingly skilled health workforce continues to grow. The demand for nursing research career pathways continues to increase in both the UK and Europe.

Challenges for the 21st century include the need for further development of postdoctoral career pathways, the development of evaluation models and their appli- cation to doctoral nursing education programs, and the development of robust funding for nursing research. All faculty members in doctoral education programs face heavy teaching loads and there is a lack of nursing research career pathways for faculty members in higher education. Finally, there is a need to develop routes that will fast-track younger nurses into research training.

\section{Japan}

Professors Shigeko Horiuchi, Dean at St Luke's College of Nursing in Tokyo, and SeonAe Yeo, of University of North Carolina at Chapel Hill in the USA, jointly presented on nursing education in Japan. Professor Horiuchi set the context for nursing and health care in Japan by examining the demographics of the society and the changing lifestyles of the citizens, which are increasing the rate of chronic diseases. In addition, the health workforce shortages and the maldistribution of healthcare resources is resulting in increasing health disparities by economic status and geographical area. These dynamics present special challenges for doctoral education in nursing, in terms of the need for increased numbers, as well as the implications for nursing science generated by the research of graduates.

Doctoral education began in Japan in 1988 and, since that time, there has been a rapid growth in programs. A recent study of 12 programs revealed that they have produced $\sim 256$ graduates during that time. The research of the graduates addresses all areas of nursing science and education.

Professor Yeo discussed her experiences in bridging two cultures and receiving education in both Japan and the USA. Both presenters examined the pressing challenges faced by doctoral programs. One critical area is how to allocated limited resources for research and how to best focus that research in a way that meets the pressing health needs of the society. Another challenge deals with how to change current clinical practise and inform health policy based on the evidence that results from the research of new doctoral graduates and their faculty members. Both presenters emphasized the importance of international collaboration in addressing the growing needs in both nursing doctoral education and nursing science globally.

\section{Thailand}

Professor Wichit Srisuphan, from Chiang Mai University in Thailand, presented the country-wide model developed in Thailand to meet the needs of doctoral education in nursing. The first doctoral program was 
established in public health nursing in 1984. Next, a collaborative doctoral program in nursing science was established among four universities in 1987. In 1998, Thailand established the first international collaborative program in nursing doctoral education, where Thai doctoral students spend 1 year of their studies abroad in a well-established doctoral program to refine their research focus and to work with a well-established research mentor in the student's area of research interest. After that year abroad, students return to their home university in Thailand to carry out their dissertation research. The student's doctoral supervision committee is comprised of faculty members from the Thai university, as well as the international mentor with which the student has studied.

Through extensive national planning, Thailand has developed seven nursing doctoral programs. They also have established country-wide goals for the number of doctorally prepared faculty members that will be needed to meet national needs. Doctoral program enrollment levels are based on this model.

\section{SUMMARY}

Several themes were evident throughout the various presentations. One is the growing international crisis that relates to health workforce shortages and the aging of the workforce. This has major implications for nursing doctoral education, in terms of the growing need for expanding the number of doctoral programs and the increased need for more nurses to be prepared at the doctoral level. A second theme relates to the level of research training needed in doctoral education programs as new graduates develop their research programs. The training programs need to be of sufficient rigor to ensure that new nurse scientists are able to address the pressing health problems faced globally and translate the findings of their research into practise and health policy. Finally, the theme of making sure that nurses are engaged in the health and social policy arena was emphasized by several presenters. It is essential that present and future doctorally prepared nurses see this as part of their roles and responsibilities.

At the conclusion of the INDEN biennial meeting, a day-long workshop was given for doctoral students. This workshop addressed how to develop and strengthen research programs, particularly after students finish their doctoral program and assume their first position as a doctorally prepared nurse scientist. This workshop was highly successful and illustrated the important role that the INDEN can play in promoting the development and strengthening of doctoral nursing education through global collaboration.

The INDEN website contains material on the scientific sessions, which can be accessed at http://www. umich.edu/ inden/. The INDEN will meet again in 2009 in South Africa in conjunction with the ICN meetings. Specific details will be available on the INDEN website as they are developed.

\section{APPENDIX I}

\section{International Network for Doctoral Education in Nursing scientific program: Critical challenges in nursing doctoral education for the 21 st century}

Scientific session: Part I (26 May 2007)

1 Needs for doctorally prepared nurses in a global context: the view from the World Health Organization (WHO)

Jean Yan, PhD, RN

Chief Scientist, Nursing and Midwifery, WHO, Geneva, Switzerland

2 Critical challenges for doctoral education in Africa Professor Hester Klopper

North-West University, South Africa

3 Employment opportunities for doctoral graduates in Australia, New Zealand, and the surrounding region Professor Kim Usher

School of Nursing, Midwifery and Nutrition, James Cook University, Australia

4 Respondent panel:

Patricia Davidson, PhD, RN

University of Western Sydney, Australia

Mi Ja Kim, PhD, RN

University of Illinois, Chicago, USA

Scientific session: Part II (27 May 2007)

1 Supplying the demand for doctoral education in nursing in the UK and Europe

Professor Anne Marie Rafferty

Florence Nightingale School of Nursing and Midwifery,

King's College London, UK

2 Development, trends, and challenges of doctoral education in Japan

Professor Shigeko Horiuchi

Dean, St Luke's College of Nursing, Japan

3 Personal reflection between two worlds from the viewpoint of advancement of nursing knowledge Professor SeonAe Yeo 
School of Nursing, University of North Carolina at Chapel Hill, USA

4 Development of a supply and demand model for nursing in Thailand

Professor Wichit Srisuphan

Faculty of Nursing, Chiang Mai University, Thailand
5 Respondent panel:

Professor Lorraine Ellis

University of Sheffield, UK

Dean Kathleen Potempa

University of Michigan, USA 